\title{
MAGNETOSTATIC ANALYSIS OF A PINCH MODE MAGNETORHEOLOGICAL VALVE
}

\author{
Janusz GOŁDASZ ${ }^{* * *}$, Bogdan SAPIŃSKI"** \\ *Cracow University of Technology, ul. Warszawska 24, 31-155 Kraków, Poland \\ *Technical Center Kraków, BWI Group, ul. Podgórki Tynieckie 2, 30-399 Kraków, Poland \\ ${ }^{* * *}$ AGH University of Science and Technology, al. Mickiewicza 30, 30-059 Kraków, Poland \\ janusz.goldasz@bwigroup.com, deep@agh.edu.pl
}

received 15 May 2017, revised 20 September 2017, accepted 22 September 2017

\begin{abstract}
The study deals with the pinch mode of magnetorheological (MR) fluids' operation and its application in MR valves. By applying the principle in MR valves a highly non-uniform magnetic field can be generated in flow channels in such a way to solidify the portion of the material that is the nearest to the flow channel's walls. This is in contrary to well-known MR flow mode valves. The authors investigate a basic pinch mode valve in several fundamental configurations, and then examine their magnetic circuits through magnetostatic finiteelement (FE) analysis. Flux density contour maps are revealed and basic performance figures calculated and analysed. The FE analysis results yield confidence in that the performance of MR pinch mode devices can be effectively controlled through electromagnetic means.
\end{abstract}

Key words: MR Fluid, Pinch Mode, Valve, Magnetostatic Model

\section{INTRODUCTION}

MR effect is manifested by changes in the material's apparent viscosity when it is exposed to magnetic field of sufficient magnitude (Jolly et al., 1996). The changes are controllable, fast and reversible. The feature has made the material attractive for use in vibration damping applications in particular. By considering the manner in which the material is operated, MR fluid based devices can be classified into four categories: flow-mode (Gołdasz and Sapiński, 2015, Simms et al., 2001, Yao et al., 2002), shear mode (Wereley et al., 2007), squeeze mode (Boelter and Janocha, 1998, Jolly and Carlson, 1996), pinch mode (Goncalves and Carlson, 2009). Hybrid devices utilize more than one operating mode of MR fluids (Yazid et al., 2014).

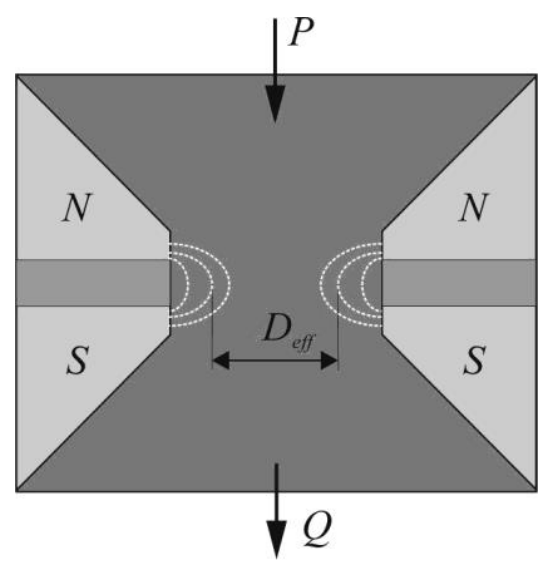

Fig. 1. Pinch mode valve concept (Goncalves and Carlson, 2009)

In this paper the so-called pinch mode is of interest. The recent invention of pinch mode valves as shown in Fig. 1 (Carlson et al., 2007) utilizes flow channels in which MR fluid is energized by a highly non-uniform magnetic field. The pressure at the inlet is $P$, and by varying the effective diameter Deff the inventors claimed the flow rate through the valve $Q$ can be adjusted. The valve features a non-magnetic spacer that separates the magnetic poles. Hence, the magnetic flux is directed in parallel to the fluid flow direction. According to the inventors the valve can be operated in a manner similar to that of a controllable orifice via field induced yield stress modifications - the material is solidified near the walls of the channel it flows through. Effectively, most of the fluid flow may occur the relatively narrow zone in the middle of the flow channel-see Fig. 2. In the figure the areas with little or no flow are characterized by high yield stresses. However, the pinch mode devices have received the least research attention, and available data are scarce (Carlson et al., 2007, Goncalves and Carlson, 2009).



Fig. 2. Simulated MR fluid flow between parallel plates and with high yield stress zones near channel walls

The purpose of this paper was to examine the steady-state performance of an MR valve that can be operated in pinch mode. The authors highlight several configurations of this valve, then proceed with a FE magnetostatic study of this device's model at various levels of the control coil current. The obtained results are then presented in the form of flux density contour maps and averaged flux density vs. current characteristics, respectively. 


\section{MR VALVE}

The pinch mode valve is illustrated in Fig. 3 . In the figure the numeric symbols indicate the key components of the valve. The valve is axi-symmetric. The core (1) houses the coil (2), the flow channel (3) and the non-magnetic spacer (4). The current in the coil induces the magnetic flux in the core assembly that passes the core, and is then directed into the flow channel. With this spacer-core-fluid arrangement the material is likely to solidify at areas near the flow gap walls. Contrary to flow mode valve, in pinch mode valves the flux passes the flow gap in the direction parallel to the fluid flow.



Fig. 3. Pinch mode valve schematic layout: 1 - core, 2 - coil, 3 - flow channel, 4 - non-magnetic spacer

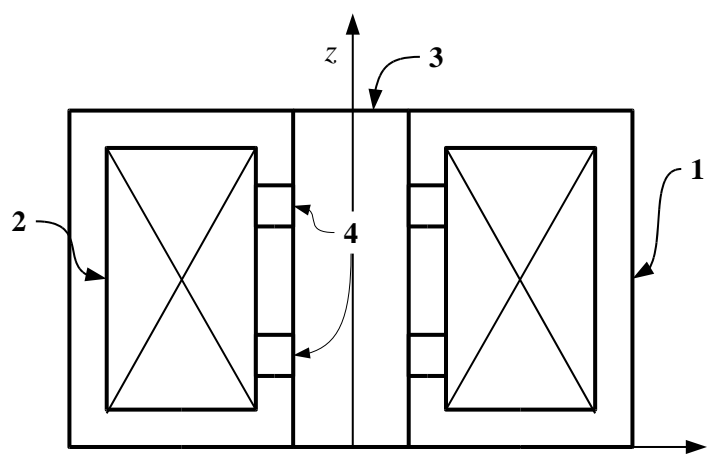

Fig. 4. Pinch mode serial valve schematic layout: 1 - core, 2 - coil, 3 - flow channel, 4 - non-magnetic spacers

In detail, the illustrated control valve height is $20 \mathrm{~mm}$, and its outer diameter is $21 \mathrm{~mm}$. The flow gap outer diameter is $4 \mathrm{~mm}$. The non-magnetic spacer length is $2 \mathrm{~mm}$. The control coil window size is $4 \times 14 \mathrm{~mm}$ (width $\mathrm{x}$ height), and it incorporates $\mathrm{N}=150$ turns of $0.51 \mathrm{~mm}$ dia. copper wire. The core material is low carbon steel alloy of the SAE 1010 grade, and the MR fluid's material characteristics are that of $26 \% \mathrm{Fe}$ vol. Following Carlson et al. (2007), the core assembly may contain one coil or more control coils in series or parallel. Utilizing multiple spacers in the flow gap simply increases the activation area, and the effective output range. Such exemplary configuration is shown in Fig. 4.

The configuration revealed in Fig. 3. incorporates three $2 \mathrm{~mm}$ long spacers to influence the magnetic field distribution in the flow gap. Again, the length of the spacing sections is $2 \mathrm{~mm}$ each. The coil and the core assemblies are identical with the design of Fig. 3. Several other configurations of such MR valve were highlighted by Carlson et al. (2009) and the reader should refer to their patent application for further details.

\section{MAGNETOSTATIC ANALYSIS}

In this section the valve's geometry is subject to an FE analysis of the magnetic circuit of the valve. The analysis is extended to the configurations of MR valve incorporating multiple serial coils and/or multiple spacers.

First, the valve's geometry highlighted in Fig. 3 was basis for the axi-symmetric 2D (two-dimensional) model developed in FEMM ver. 4.2. The obtained results were shown in Figs. 4 through 10. Specifically, the flux density variation in the control gap area directly above the non-magnetic spacer is revealed in Fig. 5. In this figure ' 0 ' denotes the gap's centreline, and '2' refers to the inner diameter of the flow channel. The results were computed for the coil ampere turns (NI) range from $150 \mathrm{AT}$ to $750 \mathrm{AT}$. For example, the figure shows that the flux density varies from appr. $0.68 \mathrm{~T}$ to $0.82 \mathrm{~T}$ for the highest excitation input of $750 \mathrm{AT}$. Moreover, the simulations show that the normal component of the flux density vector is nearly 0 , and the observed contribution is due to the tangential component only. Next, by averaging the flux density distribution in the region above the spacer the flux density vs. coil ampere turns characteristics in Fig. 6 could be obtained. It is then apparent that using the above configuration of Fig. 3 the control gap's flux density can be effectively manipulated by varying the control coil's current input level. Finally, flux density maps in Fig. 7 reveal that the highest magnetic flux concentration in the flow channel is directly in the area above the spacer.

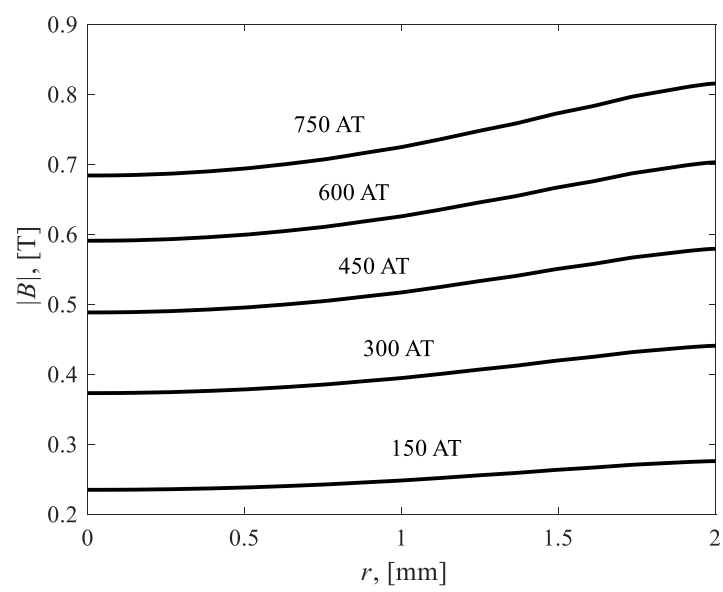

Fig. 5. Flux density variation in the flow channel



Fig. 6. Averaged flux density variation vs. coil ampere turns 

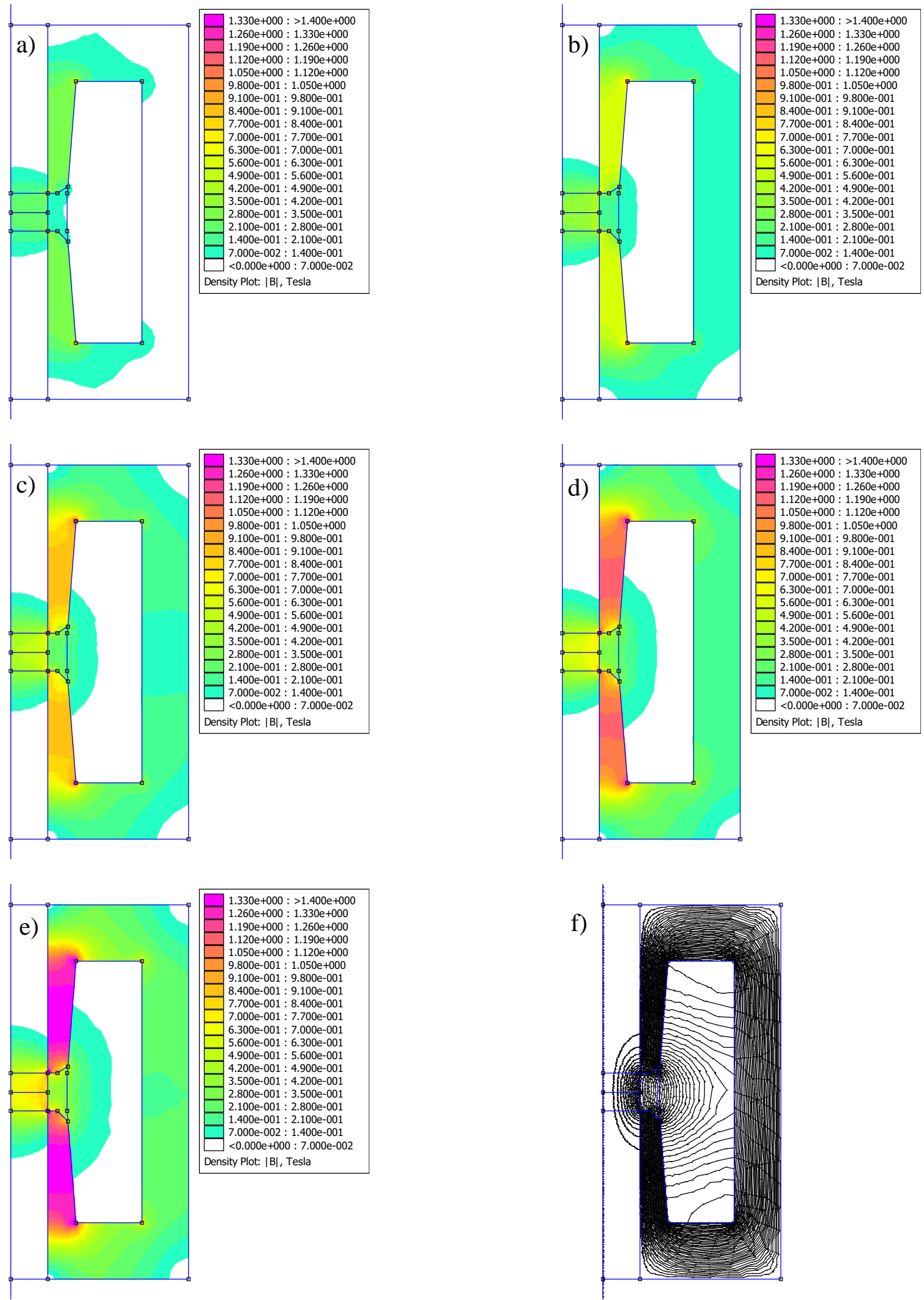

Fig. 7. Flux density contour maps and flux line distribution in single gap pinch mode, a) $150 \mathrm{AT}$, b) $300 \mathrm{AT}$, c) $450 \mathrm{AT}$, d) $600 \mathrm{AT}$, e) $750 \mathrm{AT}$, f) flux lines at $750 \mathrm{AT}$

For comparison, by analyzing the serial configuration the authors arrived at the numerical results highlighted in Fig. 8 through 10. Again, Fig. 8 illustrates flux lines distribution in the assembly, and Fig. 9 shows the averaged flux density B in the control gap vs. coil ampere turns. Specifically, the figure reveals a significantly lower effective output range for the serial configuration as the flux is required to pass through 3 air gaps. However, the activated area is 3 times larger than that of Fig. 3. Finally, Fig. 9 reveals 
averaged flux density vs. coil current calculated for a serial control gap configuration in which multiple coils were connected in series. The configuration that is illustrated in Fig. 10 features 3 coils of 50 wire turns each. The effective flux density output of this valve is almost identical to that of Fig. 9 for the same ampere turns level (750 AT total).



Fig. 8. Flux lines at $750 \mathrm{AT}$ in multiple pinch mode gaps

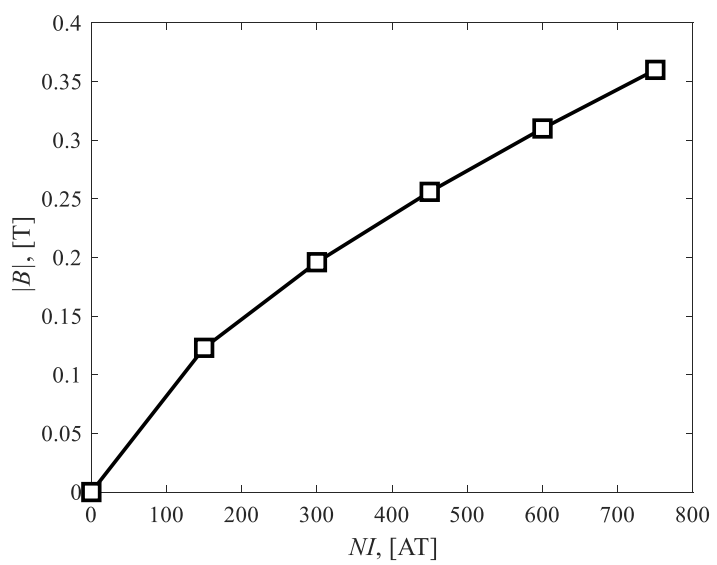

Fig. 9. Flux density vs. coil ampere turns in multiple pinch gap valve
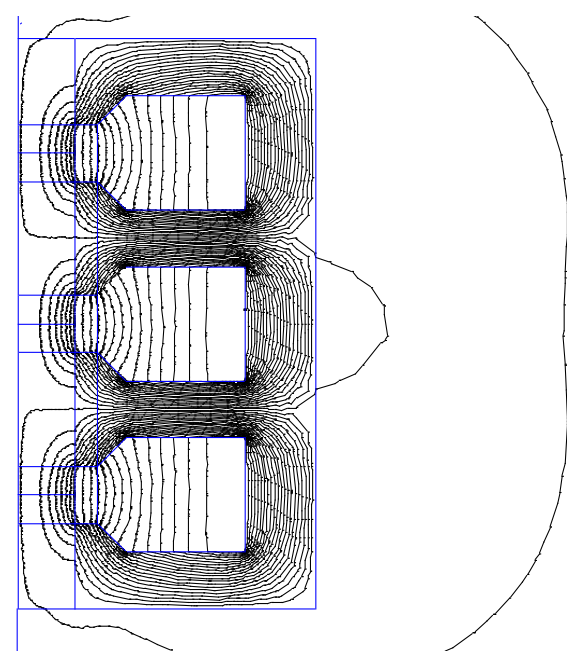

Fig. 10. Flux density map; 750 AT total in serial coil configuration
Moreover, as shown in the case of the serial coil and serial control gap configurations (see Figs. 8 and 10), respectively, the results revealed that the effective flux density is severely degraded, however, the energized surface area is significantly higher when compared against the design concept illustrated in Fig. 3.

\section{SUMMARY}

The so-called pinch-mode particular is the least examined of all operating modes of MR fluids. The amount of information available to researchers on this subject is limited, and hence the interest in such study. Goncalves and Carlson (2007) compared their valve to a controllable orifice in which the 'orifice' function was performed by the fluid solidified near the flow channel's walls rather than across the flow gap. Therefore, the authors commence their analysis by developing a magnetostatic model of a pinch mode valve with one control gap and one coil assembly, and then extend it to the analysis of a valve with serial flow gaps and serial coils. The results have shown that the output of this valve can be effectively controlled through modifications of the coil current level in the assembly. It is not known yet to what magnitude the computed performance of this valve will influence the smart fluid's rheology, and the resulting pressure vs. flow characteristics.

\section{REFERENCES}

1. Boelter R., Janocha H. (1998). Performance of long-stroke and lowstroke MR fluid dampers, 5th Annual International Symposium on Smart Structures and Materials, International Society for Optics and Photonics, 303-313.

2. Carlson J. D., Goncalves F., Catanzarite D., Dobbs D. (2007), Controllable magnetorheological fluid valve, devices, and methods, U.S. Patent Application No. 11/844, 548.

3. Gołdasz N., Sapiński B. (2015), Insight into Magnetorheological Shock Absorbers, Springer, Heidelberg.

4. Goncalves F. D., Carlson J. D. (2009), An alternate operation mode for MR fluids-magnetic gradient pinch, Journal of Physics, Conference Series, 149, 012050.

5. Jolly M. R., Bender J. W., Carlson J. D. (1999), Properties and applications of commercial magnetorheological fluids, Journal of Intelligent Material Systems and Structures, 10(1), 5-13.

6. Jolly M. R., Carlson J. D. (1996), Controllable squeeze film damping using magnetorheological fluids, Proceedings of the 5th International Conference on New Actuators, Bremen, 333-336.

7. Simms N. D., Stanway R., Johnson A. R., Mellor P. (2001), Design, testing, and model validation of an MR squeeze-flow vibration damper, SPIE's 8th Annual International Symposium on Smart Structures and Materials. International Society for Optics and Photonics, 111-120.

8. Wereley N.M., Cho J.U., Choi Y.-T., Choi S.B. (2007), Magnetorheological dampers in shear mode, Smart Materials and Structures, 17(1), 015022

9. Yao G. Z., Yap F. F., Chen G., Li W., Yeo S. H. (2002), MR damper and its application for semi-active control of vehicle suspension system, Mechatronics, 12(7), 963-973.

10. Yazid I. I. M., Mazlan S. A., Kikuchi T., Zamzuri H., Imaduddin F. (2014), Design of magnetorheological damper with a combination of shear and squeeze modes, Materials and Design (1980-2015), 54, 87-95.

This work was accomplished within the scope of the programme No. E3/627/2016/DS at Cracow University of Technology. 